\title{
Densidade de estocagem no crescimento, composição e perfil lipídico corporal do jundiá
}

\author{
Stocking density in growth, composition and body lipid profile of jundiá
}

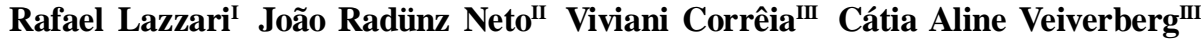 Giovani Taffarel Bergamin ${ }^{\text {IV }}$ Tatiana Emanuelli $^{\mathrm{V}}$ Cristiane Portes Ribeiro $^{\mathrm{VI}}$}

\section{RESUMO}

\begin{abstract}
O objetivo do trabalho foi avaliar o crescimento, a composição e o perfil lipídico corporal de jundiás criados em diferentes densidades de estocagem (DE: 4,2; 6,4; 8,6 e $10,8 \mathrm{~kg} \mathrm{~m}^{-3}$ ) em sistema com recirculação de água. Durante 120 dias, 420 peixes (peso inicial $=182,95 \pm 2,96 \mathrm{~g}$ ) foram alimentados uma vez ao dia, até a saciedade aparente, com dieta peletizada formulada. Observou-se que, quanto maior a $D E$ utilizada, menor é o peso individual dos peixes, entretanto a biomassa por tanque aumentou $(P<0,05)$. Melhor conversão alimentar $(1,69)$ e maior quantidade de gordura intraperitoneal $(3,92)$ foram observadas nos peixes submetidos à densidade $4,2 \mathrm{~kg} \mathrm{~m}^{-3}$. A proteína corporal foi afetada pelas DEs. Ocorreu diminuição dos ácidos graxos saturados e aumento dos insaturados nos peixes de acordo com o aumento das DEs. Conclui-se que a densidade de estocagem influência no peso final e na produtividade por volume do jundiá.
\end{abstract}

Palavras-chave: ácidos graxos, biomassa, criação, Rhamdia quelen, sistema de recirculação.

\section{ABSTRACT}

The aim of this research was to evaluate growth and body composition of jundiá farmed at different stocking densities (SD: 4.2, 6.4, 8.6 and $10.8 \mathrm{~kg} \mathrm{~m}^{-3}$ ) in a water re-use system. During 120 days, 420 fish were fed near to satiation once a day. It was observed that in the highest $S D$, the individual fish weight was lower, however the biomass per tank increased $(P<0.05)$. The best feed conversion ratio (1.69) and intraperitoneal fat (3.92\%) were observed in fish subjected to density of $4.2 \mathrm{~kg} \mathrm{~m}^{-3}$. The body protein was affected by $S D$. Saturated fatty acids decreased and unsaturated fatty acids increased according to the increase of SD. It was concluded that stocking density influences the final size and yield per volume of jundiá.

Key words: fatty acids, biomass, rearing, Rhamdia quelen, water re-use system.

\section{INTRODUÇÃO}

É imprescindível a utilização de sistemas intensivos quando se busca atender a grande demanda por carne de peixe. Apesar do elevado gasto inicial para a implantação de um sistema de recirculação e dos custos fixos com energia elétrica, este sistema requer reduzido espaço, além de permitir o reuso da água, pouca renovação, assim como maior controle dos parâmetros físicos e químicos desta água (SAMMOUTH et al., 2009).

Com a modernização dos sistemas de criação de peixes, vários fatores podem afetar significativamente a produção. Entre eles, a densidade de estocagem (DE), definida como peso por unidade de volume $\left(\mathrm{kg} \mathrm{m}^{-3}\right)$, deve ser considerada. A não

'Departamento de Zootecnia, Centro de Educação Superior Norte (CESNORS), Universidade Federal de Santa Maria (UFSM), Palmeira das Missões, RS, Brasil.

"Laboratório de Piscicultura, Departamento de Zootecnia, UFSM, 97010-120, Santa Maria, RS, Brasil. E-mail: jradunzneto@gmail.com. Autor para correspondência.

IIIPrograma de Pós-graduação em Zootecnia (PPGZ), Centro de Ciências Rurais (CCR), UFSM, Santa Maria, RS, Brasil.

${ }^{\mathrm{IV}}$ Embrapa Pesca e Aqüicultura, Palmas, TO, Brasil.

vepartamento de Tecnologia e Ciência dos Alimentos, CCR, UFSM, Santa Maria, RS, Brasil.

${ }^{\mathrm{IV}}$ Curso de Farmácia e Bioquímica, UFSM, Santa Maria, RS, Brasil. 
adaptação a altas DEs pode limitar a criação de uma espécie de peixe em condições intensivas (MOLNÁR et al., 2004).

O potencial de crescimento do peixe depende de fatores ambientais, da espécie e da DE utilizada na criação, e se espera conseguir, no menor espaço e tempo possível, maior produtividade (OUATTARA et al., 2003). As exigências nutricionais de uma espécie diferem de acordo com as instalações e as densidades de estocagem utilizadas (WEBSTER et al., 1992).

Os mecanismos relacionados ao efeito da DE no crescimento e na utilização do alimento pelos peixes são variados, como diminuição do consumo e piora na conversão alimentar (BOUJARD et al., 2002). Em muitas situações, alta DE é fator estressante para os peixes, sendo as alterações fisiológicas decorrentes as responsáveis por efeitos negativos no crescimento (VIJAYAN \& LEATHERLAND, 1988). Os efeitos de estresse provenientes de excesso de estocagem estão também atrelados à capacidade de suporte do sistema de criação, que é dependente da disponibilidade de oxigênio no interior da unidade criatória.

O jundiá (Rhamdia quelen) é um peixe nativo de hábito alimentar onívoro, com rápido crescimento e características desejáveis para o consumo, pois seus filés não possuem espinhos intramusculares (FRACALOSSI et al., 2007). A quantidade de ácidos graxos corporais em peixes é dependente de fatores fisiológicos e ambientais. ADE e a composição da dieta (MAINA et al., 2003) são exemplos de fatores ambientais. A variação das fontes lipídicas, por exemplo, interfere significativamente na composição corporal do jundiá (MELO et al., 2002). O objetivo do presente trabalho foi avaliar o crescimento e a composição corporal de jundiás submetidos a diferentes densidades de estocagem em sistema de recirculação de água.

\section{MATERIAL E MÉTODOS}

O experimento foi conduzido durante 120 dias (janeiro a abril de 2006), nas instalações do Laboratório de Piscicultura da Universidade Federal de Santa Maria - RS (altitude 95m, longitude 2943'S, latitude $\left.53^{\circ} 42^{\prime} \mathrm{W}\right)$. Para a criação dos peixes, utilizouse sistema com recirculação de água, composto por 12 tanques de amianto revestidos com fibra de vidro (850L). O sistema possui termostatos para controle da temperatura, sistema de retrolavagem, para eliminação de resíduos, e biofiltros (pedra britada) para filtragem biológica. Foram utilizados 420 jundiás sem sexagem, retirados ao acaso de um viveiro (peso inicial médio $=182,95 \pm 2,96 \mathrm{~g}$ ), distribuídos em quatro densidades de estocagem: 4,2; 6,4; 8,6 e $10,8 \mathrm{~kg} \mathrm{~m}^{-3}$ (equivalentes a $20,30,40$ e 50 peixes tanque ${ }^{-1}$ ). $\mathrm{O}$ delineamento experimental foi o inteiramente casualizado, com quatro tratamentos e três repetições.

O controle de qualidade da água foi realizado através de limpeza dos encanamentos, sifonagem diária de resíduos, renovação diária de água do sistema (10\% do volume total) e monitoramento dos parâmetros físicos e químicos: temperatura $\left({ }^{\circ} \mathrm{C}\right)$, amônia total (ppm), nitrito (ppm), oxigênio dissolvido (ppm), alcalinidade $\left(\mathrm{mg} \mathrm{CaCO}_{3} \mathrm{~L}^{-1}\right)$ e $\mathrm{pH}$. A água para as análises foi coletada na entrada do primeiro filtro biológico, antes de cada alimentação. Para aferição da temperatura, utilizou-se termômetro (bulbo de mercúrio) e, para oxigênio, oxímetro digital (YSI-Yellowsprings-EUA). Para as demais análises, foi utilizado kit colorimétricovolumétrico $\left(\right.$ Alfakit $\left.^{\circledR}\right)$. A análise de amônia foi baseada no método Indotest, e a alcalinidade por neutralização (volumétrico), ambos descritos no manual da APHA (2005). O nitrito foi analisado pelo método proposto por FRIES (1971) (método da alfa-naftilamina), e o pH pelo uso de indicador (AWWA, 1970).

A alimentação dos peixes foi realizada uma vez ao dia (10h), até a saciedade aparente. A dieta experimental foi baseada em LAZZARI et al. (2006), tendo como formulação: farinha de carne e ossos de bovinos $(23,00 \%)$, farelo de soja $(24,00 \%)$, milho moído $(23,99 \%)$, farelo de trigo $(20,00 \%)$, óleo de soja $(5,00 \%)$, sal comum $(1,00 \%)$, fosfato bicálcico $(1,00 \%)$, vitaminas e minerais $(2,00 \%)$ e antioxidante $(\mathrm{BHT})(0,01 \%)$. A dieta ofertada aos animais foi peletizada e possuía a seguinte composição bromatológica: proteína bruta $=31,09 \%$, extrato etéreo $=9,42 \%$, matéria mineral $=11,49 \%$, fibra bruta $=3,8 \%$, cálcio $=2,52 \%$ e fósforo $=1,57 \%$. A quantidade de alimento fornecido por tanque foi quantificada semanalmente por diferença de peso, para cálculo do consumo diário e conversão alimentar aparente a cada biometria (alimento oferecido (g)/ganho em peso $(\mathrm{g}))$.

Foram realizadas três biometrias para coleta de dados, no início, aos 60 e 120 dias experimentais. Os dados coletados foram peso $(\mathrm{g})$ e comprimento total (cm), utilizando balança digital com duas casas decimais e ictiômetro graduado. A partir desses valores, foram calculados: biomassa total (g), coeficiente de variação do peso $\left(\mathrm{CVP}=[\text { desvio padrão/média peso }]^{*} 100\right.$, em $\%)$, fator de condição $(\mathrm{FC}=[($ Peso x 100) $/($ Comprimento total $\left.\left.{ }^{3}\right)\right]$ ) e consumo diário aparente (CDA), em \% do peso vivo $=$ consumo no período $(\mathrm{g}) /\{($ peso final $(\mathrm{g})+$ peso inicial $(\mathrm{g})) / 2\} /$ dias $* 100$.

Antes do início do experimento, uma amostra de 20 peixes foi separada, para análises de composição centesimal inicial. Ao final do experimento, doze peixes por tratamento foram capturados e abatidos para a realização da análise de composição proximal e do perfil lipídico. Em todos os procedimentos, os peixes 
passaram por jejum (24 horas) e foram abatidos por hipotermia. As cinzas e umidade foram determinadas através da metodologia descrita nas normas analíticas do INSTITUTO ADOLFO LUTZ (1985). A gordura foi extraída e quantificada pelo método de BLIGH-DYER (1959), extração com clorofórmio/metanol, a frio. A proteína bruta foi quantificada pelo método de KJELDAHL (fator de conversão=6,25) (AOAC, 1995). Para determinação do perfil lipídico, a gordura extraída foi metilada de acordo com HARTMAN \& LAGO (1973) e analisada por cromatografia gasosa de alta resolução (cromatógrafo Hewlett-Packard, modelo HP 6890, equipado com detector de ionização de chama - FID), em coluna capilar DB-23 (Agilent -60m x 0,25mm x $0,25 \mu \mathrm{m})$.

Os dados obtidos foram submetidos ao teste de Shapiro-Wilk para verificação de normalidade. As variáveis com distribuição não normal foram submetidas à ANOVA de Kruskall-Wallis. O procedimento MIXED do software SAS ${ }^{\circledR}$ (1997) foi aplicado para avaliar o efeito do tempo (60 ou 120 dias) em cada variável resposta de desempenho. O efeito da densidade de estocagem foi avaliado por regressão polinomial $(\mathrm{P}<0,05)$.

\section{RESULTADOS E DISCUSSÃO}

A qualidade da água de criação esteve dentro de níveis aceitáveis para a criação do jundiá: temperatura $22,7 \pm 2,6^{\circ} \mathrm{C}$; amônia total $0,9 \pm 0,6 \mathrm{mg} \mathrm{L}^{-1}$; nitrito $0,1 \pm 0,1 \mathrm{mg} \mathrm{L}^{-1}$; oxigênio dissolvido $5,1 \pm 1,1 \mathrm{mg} \mathrm{L}^{-1}$; alcalinidade $32,0 \pm 6,1 \mathrm{mg} \mathrm{CaCO}_{3} \mathrm{~L}^{-1} ; \mathrm{pH} 6,7 \pm 0,3$.

$\mathrm{O}$ peso individual dos peixes diminuiu de acordo com a elevação da DE (Figura 1A), nos dois períodos de avaliação ( 60 dias: $Y=279,48-6,40 X, r^{2}=0,67$, $\mathrm{P}=0,001 ; 120$ dias: $\left.\mathrm{Y}=298,34-6,02 \mathrm{X}, \mathrm{r}^{2}=0,68, \mathrm{P}=0,003\right)$. Ao final do experimento, os peixes da DE $4,2 \mathrm{~kg} \mathrm{~m}^{-3}$ atingiram maior peso $(315 \mathrm{~g})$. Os resultados evidenciam diminuição no crescimento do jundiá em função da elevação da densidade de estocagem em sistema de recirculação de água. Para o tambaqui Colossoma macropomum, embora sendo criado em tanque-rede, com densidade variando entre 200 e 500 peixes $\mathrm{m}^{-3}$, também se verificou relação inversa entre densidade e peso corporal, sem alteração na sobrevivência (BRANDÃO et al., 2004).

A melhor DE varia de acordo com a espécie e o sistema de criação. Para a tilápia Oreochromis niloticus, criada em água marinha, em gaiolas flutuantes, o melhor desempenho foi obtido nas densidades de 5,98 a $12,00 \mathrm{~kg} \mathrm{~m}^{-3}$ (WATANABE et al., 1990) e 14,10 a 30,70 kg m (CRUZ \& RIDHA, 1989). ROWLAND et al. (2004) relatam que a variação da DE de 50 para 200 peixes $\mathrm{m}^{-3}$ (peso médio inicial de $2,3 \mathrm{~g}$ ) não afeta o crescimento e sobrevivência de perca prateada Bidyanus bidyanus, criada em tanques e em gaiolas. Entretanto, quando se comparou o sistema de criação (gaiola ou tanque de terra), verificou-se que os peixes mantidos em gaiolas flutuantes cresceram mais rapidamente que os mantidos em tanques.

Alguns trabalhos demonstram que, em maiores densidades, os peixes são mais agressivos na busca e ingestão do alimento, resultando em maior sobrevivência, sem redução de crescimento, consumo e conversão alimentar (MOLNÁR et al., 2004; ROWLAND et al., 2004). Entretanto, também podem ocorrer alterações no comportamento e pior utilização do alimento, resultando em mortalidade e crescimento reduzido (MONTERO et al., 1999).

A elevação da DE implicou maior biomassa total por tanque (Figura 1). Tanto aos 60 $\left(\mathrm{Y}=1606,57+842,35 \mathrm{x}, \mathrm{r}^{2}=0,97, \mathrm{P}=0,0001\right)$ como aos 120 $\operatorname{dias}\left(\mathrm{Y}=1560,53+951,29 \mathrm{x}, \mathrm{r}^{2}=0,96, \mathrm{P}=0,0001\right)$, o efeito da $\mathrm{DE}$ sobre a biomassa foi linear, altamente significativo. Observou-se correlação negativa $(r=-0,72, \mathrm{P}=0,007)$ entre o peso individual dos jundiás e a biomassa total. Em relação ao tempo de amostragem (Figura 1C), observou-se aumento linear significativo da biomassa em todas as densidades testadas. Observa-se, na figura, entretanto, que, nas densidades acima de $4,2 \mathrm{~kg} \mathrm{~m}^{-3}$, o crescimento dos peixes foi se estabilizando, indicando que a capacidade de suporte do sistema de criação pode ter sido atingida.

O consumo diário dos peixes apresentou redução de acordo com o aumento da DE (Tabela 1). Para algumas espécies de peixes, principalmente salmonídeos, o aumento da DE resulta em diminuição da atividade alimentar, refletido em menor consumo de ração (BAKER \& AYLES, 1990). Os jundiás das densidades 8,6 e $10,8 \mathrm{~kg} \mathrm{~m}^{-3}$ apresentaram consumo menor em relação aos demais. A diminuição do consumo de alimento em função do aumento da DE pode estar relacionada ao estresse (ALANÄRÄ \& BRÄNNÄS, 1996). Segundo ELLIS et al. (2002), o incremento da densidade de estocagem em trutas resulta em interações sociais e consequente redução do consumo, levando à diminuição do crescimento e, por consequência, do bem-estar dos peixes.

Peixes criados em baixas DEs apresentam maior crescimento individual e sobrevivência, entretanto, a produção por área é baixa (GOMES et al., 2000). O surgimento de dominância e aumento da condição de estresse são sintomas característicos de altas DEs (CAVERO et al., 2003). Neste sentido, esperase que maiores DE apresentem grandes variações entre indivíduos, como observado para a matrinxã Brycon cephalus e o black bass Micropterus dolomien (GOMES et al., 2000; PETIT et al., 2001). No presente estudo, contudo, não se verificou efeito das DEs $(\mathrm{P}>0,05)$ testadas sobre o coeficiente de variação do peso dos jundiás. 

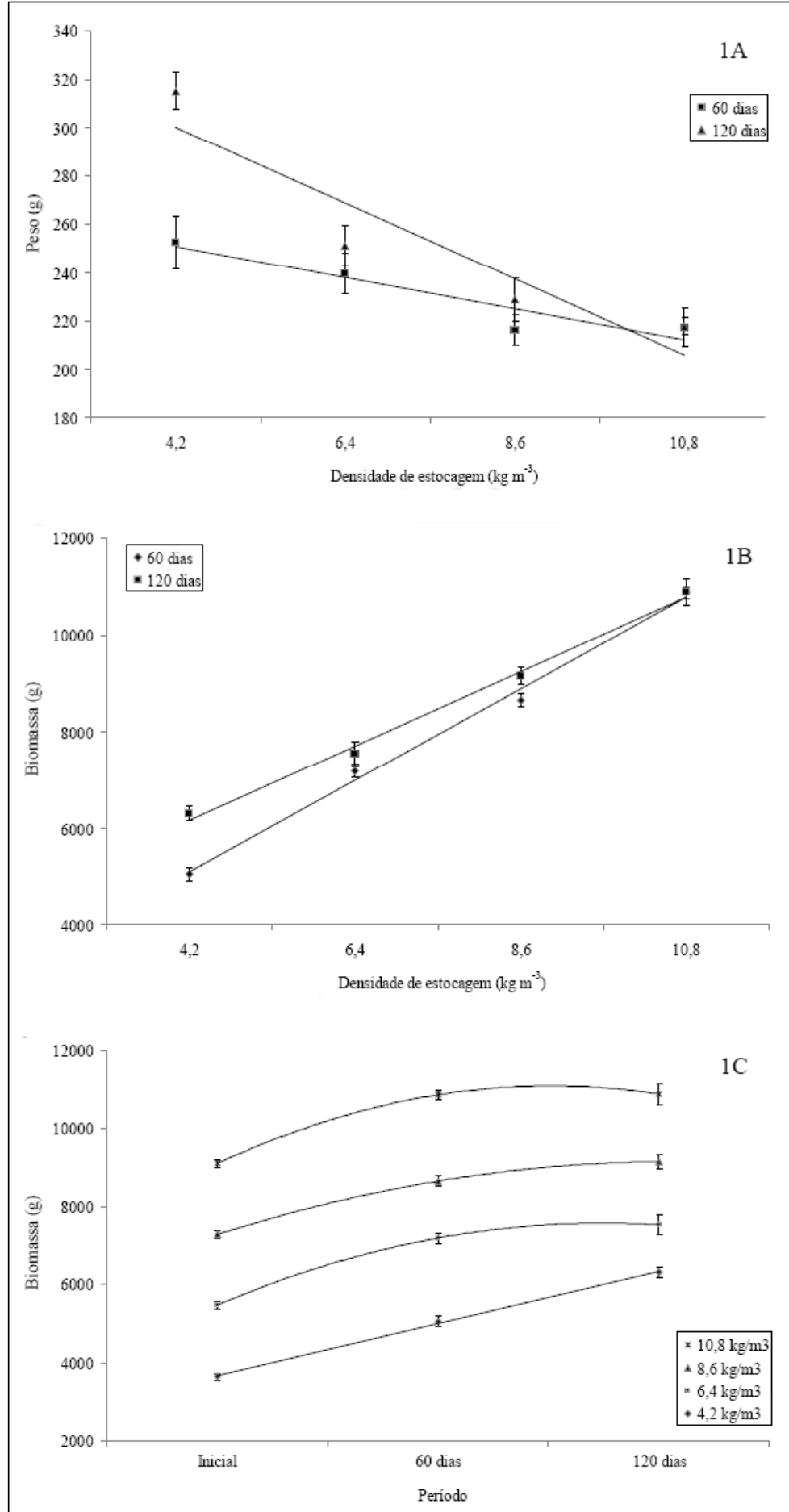

Figura 1 - Peso (1A), biomassa total em função da densidade (1B) e evolução da biomassa em relação aos tempos de amostragem (1C) de jundiás submetidos a diferentes densidades de estocagem em sistema de recirculação de água. Regressão significativa para a biomassa em todas as densidades: $4,2 \mathrm{~kg} \mathrm{~m}^{-3}: \mathrm{Y}=3820,94+15,26 \mathrm{X}, \mathrm{r}^{2}=0,85$, $\mathrm{P}=0,0003 ; 6,4 \mathrm{~kg} \mathrm{~m}^{-3}: \mathrm{Y}=5621,94+20,75 \mathrm{X}, \mathrm{r}^{2}=0,86, \mathrm{P}=0,0003 ; 8,6$ $\mathrm{kg} \quad \mathrm{m}^{3}: \mathrm{Y}=7368,44+17,92 \mathrm{X}, \quad \mathrm{r}^{2}=0,87, \quad \mathrm{P}=0,0002 ; \quad 10,8 \quad \mathrm{~kg}$ $\mathrm{m}^{3}: \mathrm{Y}=9120,72+23,73 \mathrm{X}, \mathrm{r}^{2}=0,95, \mathrm{P}<0,0001$; 
Tabela 1 - Parâmetros de desempenho de jundiás submetidos a diferentes densidades de estocagem.

\begin{tabular}{|c|c|c|c|c|c|c|}
\hline \multirow{2}{*}{ Variáveis } & \multicolumn{4}{|c|}{------------Densidade de estocagem $\left(\mathrm{kg} \mathrm{m}^{-3}\right.$} & \multirow{2}{*}{ dpr } & \multirow{2}{*}{$\mathrm{P}$} \\
\hline & 4,2 & 6,4 & 8,6 & 10,8 & & \\
\hline & \multicolumn{6}{|c|}{ 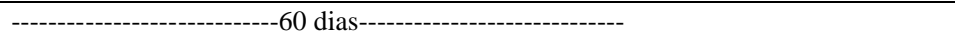 } \\
\hline Comprimento total $(\mathrm{cm})$ & 27,78 & 27,37 & 27,23 & 27,20 & 0,79 & NS \\
\hline Comprimento padrão $(\mathrm{cm})$ & 23,26 & 22,71 & 23,18 & 22,49 & 1,57 & NS \\
\hline Coeficiente de variação do peso $(\%)$ & 4,26 & 7,85 & 2,75 & 3,56 & 5,20 & NS \\
\hline Fator de condição $^{1}$ & 1,19 & 1,18 & 1,07 & 1,07 & 0,03 & $* *$ \\
\hline Consumo diário $(\% / \text { peso vivo })^{2}$ & 1,69 & 1,49 & 1,25 & 1,05 & 0,07 & $* * *$ \\
\hline Conversão alimentar aparente ${ }^{3}$ & 1,65 & 1,78 & 2,35 & 1,90 & 0,26 & $*$ \\
\hline \multirow[t]{2}{*}{ Gordura intraperitoneal $(\%)^{4}$} & 1,46 & 3,23 & 3,39 & 2,08 & 1,16 & $*$ \\
\hline & \multicolumn{6}{|c|}{ 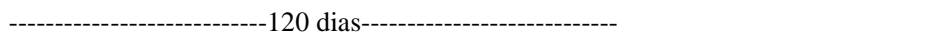 } \\
\hline Comprimento total $(\mathrm{cm})$ & 28,62 & 28,33 & 27,81 & 28,08 & 0,96 & NS \\
\hline Comprimento padrão $(\mathrm{cm})$ & 23,95 & 23,58 & 23,16 & 23,18 & 0,49 & NS \\
\hline Coeficiente de variação do peso $(\%)$ & 10,32 & 16,29 & 13,90 & 14,66 & 7,30 & NS \\
\hline Fator de condição ${ }^{5}$ & 1,16 & 1,14 & 1,08 & 1,05 & 0,02 & $* * *$ \\
\hline Consumo diário (\%/peso vivo $)^{6}$ & 1,38 & 1,50 & 1,17 & 1,04 & 0,11 & $* *$ \\
\hline Conversão alimentar aparente ${ }^{7}$ & 1,69 & 3,08 & 3,30 & 3,72 & 0,32 & $* * *$ \\
\hline Gordura intraperitoneal $(\%)^{8}$ & 3,92 & 3,30 & 2,57 & 1,88 & 1,68 & $*$ \\
\hline
\end{tabular}

$* \mathrm{P}<0,05 ; * * \mathrm{P}<0,01 ; * * * \mathrm{P}<0,0001$. NS: Não significativo em um nível mínimo de $5 \%$.

dpr=desvio padrão residual.

${ }^{1} \mathrm{Y}=1,28-0,02 \mathrm{X}, \mathrm{r}^{2}=0,68 ;{ }^{2} \mathrm{Y}=2,10-0,09 \mathrm{X}, \mathrm{r}^{2}=0,93 ;{ }^{3} \mathrm{Y}=-0,03+0,51 \mathrm{X}-0,03 \mathrm{X}^{2}, \mathrm{r}^{2}=0,45 ;{ }^{4}$ Significativo pela ANOVA de Kruskall-Whalis (não paramétrica); ${ }^{5} \mathrm{Y}=1,24-0,01 \mathrm{X}, \mathrm{r}^{2}=0,91 ;{ }^{6} \mathrm{Y}=1,74-0,06 \mathrm{X}, \mathrm{r}^{2}=0,69 ;{ }^{7} \mathrm{Y}=0,79+0,28 \mathrm{X}, \mathrm{r}^{2}=0,78 ;{ }^{8} \mathrm{Y}=5,25-0,31 \mathrm{X}, \mathrm{r}^{2}=0,40$.

Para o fator de condição (FC), verificou-se efeito linear decrescente das DE, nos dois períodos de avaliação (60 e 120 dias) (Tabela 1). Isto confirma os resultados de peso individual, podendo-se inferir que o jundiá submetido a maiores densidades apresenta pior desempenho. Os dados de conversão alimentar aparente (CAA) dos peixes (Y) em função da DE (X), ajustam-se a uma quadrática $\left(\mathrm{Y}=-0,03+0,51 \mathrm{x}-0,03 \mathrm{x}^{2}\right.$, $\left.\mathrm{r}^{2}=0,45, \mathrm{P}<0,05\right)$, nos 60 dias experimentais. Aos 120 dias, a CAA dos jundiás aumentou de acordo com a $\mathrm{DE}$, ajustando-se a uma equação linear $(\mathrm{Y}=0,79+0,28 \mathrm{x}$, $\left.\mathrm{r}^{2}=0,78, \mathrm{P}=0,0001\right)$. Interações comportamentais ocorrem principalmente em altas densidades, nas quais geralmente o peixe destina parte de suas reservas para suportar uma situação desfavorável oriunda de alguma situação de estresse (IRWIN et al., 1999). Ressalta-se a redução da quantidade de gordura intraperitoneal (Y) em função da elevação da DE (X), ajustada a uma equação linear ( $\left.Y=5,25-0,31 \mathrm{x}, \mathrm{r}^{2}=0,40\right)$ (Tabela 1$)$.

Em relação aos teores de proteína do peixe inteiro (Tabela 2), observou-se ajuste a uma curva quadrática da DE. Os demais dados de composição proximal da carcaça não foram afetados pela DE. Os valores de composição encontrados neste estudo são similares aos encontrados em outros trabalhos com a espécie, demonstrando que a DE também pode influenciar a variação dos componentes corporais, além da composição da dieta, já comprovada por LAZZARI et al. (2006).
Quanto ao perfil lipídico corporal dos jundiás (Tabela 2), observou-se maior quantidade de ácidos graxos saturados nos peixes submetidos à menor densidade testada $\left(4,2 \mathrm{~kg} \mathrm{~m}^{-3}\right)$. Dos AGs insaturados, o único que foi afetado pela $\mathrm{DE}$ foi o C22:5n-3, ocorrendo em maior quantidade nas duas maiores densidades testadas $(P<0,01)$. Foi verificado aumento linear $\left(\mathrm{Y}=64,12+0,07 \mathrm{x}, \mathrm{r}^{2}=0,4\right)$ na quantidade total de ácidos graxos insaturados (Y) em função da elevação da DE (X) $(\mathrm{P}<0,05)$. As relações n-3/n-6 e também entre os AGs insaturados/AGs saturados também foram maiores nas DEs mais altas $(\mathrm{P}<0,05)$.

\section{CONCLUSÃO}

A elevação da densidade de estocagem de 4,2 para $6,4 \mathrm{~kg} \mathrm{~m}^{-3}$ proporciona redução no peso individual, aumento da biomassa e diminuição nos teores de ácidos graxos saturados do jundiá. A proteína corporal e o perfil lipídico são afetados pela densidade de estocagem.

\section{AGRADECIMENTOS}

À SEAP/MCT/FINEP/Fundo Setorial Agronegócio/ Fundo CT Hidro-Aquicultura (Processo n.3602/05) pelo auxílio financeiro ao trabalho. À Coordenação de Aperfeiçoamento de Pessoal de Nível Superior (CAPES), pela concessão de bolsa de estudos, ao Conselho Nacional de Desenvolvimento Científico e Tecnológico ( $\mathrm{CNPq}$ ), pelas bolsas de produtividade em pesquisa 
Tabela 2 - Composição e perfil lipídico corporal de jundiás submetidos a diferentes densidades de estocagem ao final do experimento.

\begin{tabular}{|c|c|c|c|c|c|c|}
\hline \multirow[b]{2}{*}{ Constituintes (\%) } & \multicolumn{6}{|c|}{----------Densidade de estocagem $\left(\mathrm{kg} \mathrm{m}^{-3}\right)$---------- } \\
\hline & 4,2 & 6,4 & 8,6 & 10,8 & $\mathrm{dpr}$ & $\mathrm{P}$ \\
\hline & \multicolumn{6}{|c|}{ Composição corporal------ } \\
\hline Umidade & 69,30 & 67,60 & 70,55 & 68,80 & 1,94 & NS \\
\hline Cinzas & 2,49 & 2,90 & 2,75 & 3,16 & 0,46 & NS \\
\hline Proteína $^{1}$ & 17,41 & 16,23 & 15,29 & 17,54 & 0,96 & * \\
\hline Lipídios & 12,55 & 13,23 & 10,95 & 10,80 & 1,57 & NS \\
\hline Ácidos graxos (\% nos lipídios totais) & \multicolumn{6}{|c|}{ 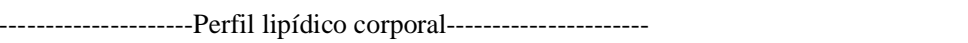 } \\
\hline $\mathrm{C} 14: 0^{2}$ & 1,48 & 1,54 & 1,45 & 1,30 & 0,11 & $* *$ \\
\hline $\mathrm{C} 16: 0^{3}$ & 24,34 & 22,84 & 23,37 & 22,10 & 1,08 & $*$ \\
\hline C18:0 & 8,57 & 8,14 & 7,93 & 8,12 & 0,44 & NS \\
\hline$\sum$ Saturados $(\mathrm{SFA})^{4}$ & 34,87 & 33,12 & 33,20 & 32,26 & 1,24 & $*$ \\
\hline C16:1n-7c & 5,69 & 6,40 & 6,30 & 5,61 & 0,78 & NS \\
\hline C18:1n-9c & 35,47 & 35,53 & 35,75 & 36,48 & 0,79 & NS \\
\hline C20:1n-9 & 0,90 & 0,94 & 0,68 & 1,09 & 0,31 & NS \\
\hline$\Sigma$ Monoinsaturados & 42,15 & 42,97 & 42,81 & 43,24 & 1,50 & NS \\
\hline$C 18: 2 n-6 c$ & 18,97 & 19,76 & 19,33 & 19,66 & 1,04 & NS \\
\hline C18:3n-3 & 1,34 & 1,32 & 1,35 & 1,61 & 0,27 & NS \\
\hline C20:4n-6 & 1,07 & 1,15 & 1,29 & 1,23 & 0,27 & NS \\
\hline$C 22: 5 n-3^{5}$ & 0,29 & 0,33 & 0,44 & 0,39 & 0,07 & $* *$ \\
\hline C22:6n-3 & 1,29 & 1,32 & 1,55 & 1,57 & 0,20 & NS \\
\hline$\Sigma$ Poli-insaturados (PUFA) & 22,97 & 23,90 & 23,99 & 24,49 & 1,58 & NS \\
\hline$\sum$ Insaturados (UFA) ${ }^{6}$ & 65,13 & 66,87 & 66,79 & 67,73 & 1,24 & $*$ \\
\hline$\sum \mathrm{n}-3$ & 2,93 & 2,98 & 3,36 & 3,58 & 0,43 & NS \\
\hline$\sum \mathrm{n}-6$ & 20,04 & 20,91 & 20,63 & 20,90 & 1,24 & NS \\
\hline Relação n-3/n- $6^{7}$ & 0,14 & 0,14 & 0,16 & 0,17 & 0,01 & $*$ \\
\hline UFA/SFA ${ }^{8}$ & 1,87 & 2,02 & 2,01 & 2,10 & 0,10 & $*$ \\
\hline
\end{tabular}

$* \mathrm{P}<0,05 ; * * \mathrm{P}<0,01 ; \mathrm{NS}$ : Não significativo a um nível mínimo de $5 \%$. dpr=desvio padrão residual.

${ }^{1} \mathrm{Y}=25,72-2,68 \mathrm{X}+0,18 \mathrm{X}^{2}, \mathrm{r}^{2}=0,62 ;{ }^{2} \mathrm{Y}=1,69-0,07 \mathrm{X}, \mathrm{r}^{2}=0,51 ;{ }^{3}$ Significativo pela ANOVA de Kruskall-Whalis (não paramétrica); ${ }^{4} \mathrm{Y}=35,87-$ $0,07 \mathrm{X}, \mathrm{r}^{2}=0,56 ;{ }^{5} \mathrm{Y}=-0,07+0,02 \mathrm{X}-0,0002 \mathrm{X}^{2}, \mathrm{r}^{2}=0,43 ;{ }^{6} \mathrm{Y}=64,12+0,07 \mathrm{X}, \mathrm{r}^{2}=0,40 ;{ }^{7} \mathrm{Y}=0,11+0,001 \mathrm{X}, \mathrm{r}^{2}=0,43 ;{ }^{8} \mathrm{Y}=1,78+0,006 \mathrm{X}, \mathrm{r}^{2}=0,40$.

e de iniciação científica (PIBIC-UFSM) e à Fundação de Amparo à Pesquisa do Estado do Rio Grande do Sul (FAPERGS) pela bolsa de iniciação científica. Às empresas Frigorífico Silva e Vitagri Nutrição Animal pelo fornecimento de ingredientes e análise da dieta.

\section{REFERÊNCIAS}

ALANÄRÄ, A.; BRÄNNÄS, E. Dominance in demand feeding behavior in Arctic char and rainbow trout: the effect of stocking density. Journal of Fish Biology, v.48, p.242-254, 1996. Disponível em: <http://onlinelibrary.wiley.com/doi/10.1111/ j.1095-8649.1996.tb01116.x/pdf>. Acesso em: 20 jul. 2009. doi: $10.1111 / \mathrm{j} .1095-8649.1996 . t b 01116 . x$.

AOAC (Association of Official Analytical Chemists). Official methods of analysis of AOAC. 16.ed. Washington, DC: Patricia Cunniff, 1995. 1141p.

APHA (American Public Health Association). EATON, A.D. et al. (Eds.). Standard methods for the examination of water and wastewater. 21.ed. Washington, DC, 2005. 1368p.

AWWA (American Water Works Association). Processos simplificados para exame e análise da água. São Paulo, 1970. 276p.
BAKER, R.F.; AYLES, G.B. The effects of varying density and loading level on the growth of Arctic charr (Salvelinus alpinus) and rainbow trout (Oncorhynchus mykiss). World Aquaculture, (magazine), v.21, p.58-63, 1990.

BLIGH, E.G.; DYER, W.J. A rapid method of total lipid extraction and purification. Canadian Journal of Biochemistry and Physiology, v.37, p.911-917, 1959.

BOUJARD, T. et al. Feeding behavior, energy expenditure and growth of rainbow trout in relation to stocking density and food accessibility. Aquaculture Research, v.33, p.1233-1242, 2002. Disponível em: <http://onlinelibrary.wiley.com/doi/ 10.1046/j.1365-2109.2002.00755.x>. Acesso em: 12 ago. 2009. doi: 10.1046/j.1365-2109.2002.00755.x.

BRANDÃO, F.R. et al. Densidade de estocagem de juvenis de tambaqui durante a recria em tanques-rede. Pesquisa Agropecuária Brasileira, v.39, p.357-362, 2004. Disponível em: <http://www.scielo.br/ scielo.php?script=sci_arttext \&pid=S0100204X2005000300014\&lng =pt\&nrm=iso>. Acesso em: 01 set. 2009. doi: 10.1590/S0100204X2005000300014.

CAVERO, B.A.S. et al. Efeito da densidade de estocagem na homogeneidade do crescimento de juvenis de pirarucu em ambiente confinado. Pesquisa Agropecuária Brasileira, 
v.38, p.103-107, 2003. Disponível em: <http://www.scielo.br/ scielo.php?script=sci_arttext\&pid=S0100204X2003000100014\&ln $\mathrm{g}=\mathrm{pt} \& \mathrm{nrm}=\mathrm{iso}>$. Acesso em: 30 ago. 2009. doi: 10.1590/ S0100-204X2003000100014.

CRUZ, E.M.; RIDHA, M. Preliminary study on the production of the tilapia, Oreochromis spilurus (Gunther), cultured in sea water cages. Aquaculture Research, v.20, p.381-388, 1989. Disponível em: <http://onlinelibrary.wiley.com/doi/10.1111/ j.1365-2109.1989.tb00365.x. Acesso em: 20 jul. 2009. doi: 10.1111/j.1365-2109.1989.tb00365.x.

ELLIS, et al. The relationships between stocking density and welfare in farmed Rainbow trout. Journal of Fish Biology, v.61, p.493531, 2002. Disponível em: <http://onlinelibrary.wiley.com/doi/ 10.1111/j.1095-8649.2002.tb00893.x>. Acesso em: 12 jul. 2009. doi: 10.1006/jfbi.2002.2057.

FRACALOSSI, D.M. et al. O mito da onivoria do jundiá. Panorama da Aqüicultura, v.17, p.36-40, 2007.

FRIES, J. Análisis de trazas: métodos fotométricos comprobados. Darmstadt: Merck, 1971. 184p.

GOMES, L.C. et al. Effect of stocking density on water quality, survival, and growth of larvae of matrinxã, Brycon cephalus (Characidae), in ponds. Aquaculture, v.183, p.7381, 2000. Disponívelem: <http://www. sciencedirect.com/ science_sk $=998169998 \& \mathrm{wchp}=\mathrm{dGLbVlWzSkWA} \& \mathrm{md} 5=\mathrm{d} 5$ 9c9ccdafab71621a0567bdb0be6e33\&ie=/sdarticle.pdf $>$. Acesso em: 20 ago. 2009. doi: 10.1016/S00448486(99)00288-4.

HARTMAN, L.; LAGO, B.C.N. A rapid preparation of fatty methyl esters from lipids. Laboratory Practice, v.22, p.475477, 1973.

INSTITUTO ADOLFO LUTZ. Normas analíticas do Instituto Adolfo Lutz. V.1 - Métodos químicos e físicos para análise de alimentos. São Paulo, 1985. 368p.

IRWIN, S. et al. Stocking density, growth and growth variation in juvenile turbot, Scophthalmus maximus (Rafinesque). Aquaculture, v.178, p.77-88, 1999. Disponível em: <http://www.sciencedirect.com/ science?_ob=ArticleURL\&_udi=B6T4D3WRJKCT6\&_bd3263c143cb0510dd07 $18 f 9 f 5 \mathrm{~d} 37926 \&$ searchtype $=a>$. Acesso em: 02 set. 2009. doi:10.1016/ S0044-8486(99)00122-2.

LAZZARI, R. et al. Diferentes fontes protéicas para a alimentação do jundiá (Rhamdia quelen). Ciência Rural, v.36, p.240-246, 2006. Disponível em: <http://www.scielo.br/ scielo.php?script=sci_arttext\&pid=S010384782006000100037\&lng $=$ pt\&nrm=iso $>$. Acesso em: 12 jul. 2009. doi: 10.1590/S010384782006000100037.

MAINA, J.G. et al. Partial replacement of fishmeal with sunflower cake and corn oil in diets for tilapia Oreochromis niloticus (Linn): effect on whole body fatty acids. Aquaculture Research, v.34, p.601-608, 2003. Disponível em: <http:// onlinelibrary.wiley.com/doi/10.1111/are.2003.34.issue-8/ issuetoc>. Acesso em: 8 dez. 2010. doi: 10.1046/j.13652109.2003.00848.x.

MELO, J.F.B. et al. Desenvolvimento e composição corporal de alevinos de jundiá (Rhamdia quelen) alimentados com dietas contendo diferentes fontes de lipídios. Ciência Rural, v.32, n.2, p.323-327, 2002. Disponível em: <http://www.scielo.br/ scielo.php?script $=$ sci_arttext\&pid $=$ S0 10384782002000200023 \&lng=pt\&nrm=iso >. Acesso em: 8 set. 2009. doi: 10.1590/ S0103-84782002000200023.
MOLNÁR, T. et al. The effect of initial stocking density on growth and survival of pike-perch fingerlings reared under intensive conditions. Aquaculture International, v.12, p.181189, 2004. Disponível em: <http://www.springerlink.com/ content/x642g7g622418759/>. Acesso em 12 ago. 2009. doi: 10.1023/B:AQUI.0000032079.62056.8c.

MONTERO, D. et al. High stocking density produces crowding stress altering some physiological and biochemical parameters in gilthead seabream, Sparus aurata, juveniles. Fish Physiology and Biochemistry, v.20, p.53-60, 1999. Disponível em: <http:/ /www.springerlink.com/content/v319431545370616/>. Acesso em: 04 set. 2009. doi: 10.1023/A:1007719928905.

OUATTARA, N.I. et al. Aquaculture potential of the black-chinned tilapia, Sarotherodon melanotheron (Cichlidae). Comparative study of the effect of stocking density on growth performance of landlocked and natural populations under cage culture conditions in Lake Ayame. Aquaculture Research, v.34, p.1223-1229, 2003. Disponível em: <http://onlinelibrary.wiley.com/doi/10.1046/ j.1365-2109.2003.00921.x>. Acesso em: 22 ago. 2009. doi: 10.1046/j.1365-2109.2003.00921.x.

PETIT, G. et al. Density effects on food intake and growth of largemouth bass (Micropterus salmoides). Aquaculture Research, v.32, p.495-497, 2001. Disponível em: <http:// onlinelibrary.wiley.com/doi/10.1046/j.1365-2109.2001.00589.x>. Acesso em 15 jul. 2009. doi: 10.1046/j.1365-2109.2001.00589.x.

ROWLAND, S.J. et al. Production of silver perch (Bidyanus bidyanus) fingerlings at three stocking densities in cages and tanks. Aquaculture, v.229, p.193-202, 2004. Disponível em: <http://www.sciencedirect.com/ science?ob=ArticleURL\&_udi=B6T4D48WJRGF1\&_userid=687358\&md5=cf c21 ae90affcad4f8ae7a817fde037a\&searchtype=a.>. Acesso em 22 ago. 2009. doi: 10.1016/S0044-8486(03)00391-0.

SAMmOUTH, S. et al. The effect of density on sea bass (Dicentrarchus labrax) performance in a tank-based recirculating system. Aquacultural Engineering, v.40, n.2, p.72-78, 2009. Disponível em: <http://www.sciencedirect.com/ science?_ob=ArticleURL\&_udi=B6T4C4V17CR22\&_user=687358\&_urlVersion=0 \&_userid=687358\&md5=a7250bac9d2b059891e1717b70db2069\&searchtype=a). Acesso em: 8 dez. 2010. doi: 10.1016/j.aquaeng.2008.11.004.

SAS INSTITUTE. Statistical Analysis System. User's guide. Version 6.08. 4.ed. North Caroline, 1997. 846p.

VIJAYAN, M.M.; LEATHERLAND, J.F. Effect of stocking density on the growth and stress-response in brook charr, Salvelinus fontinalis. Aquaculture, v.75, p.159-170, 1988. Disponível em: <http:// www.sciencedirect.com/science?_ob=ArticleURL\&_udi=B6T4D49NPJKSTK\&_user $=687358 \& \_$coverDate $=12 \% 2 \mathrm{~F} 01 \% 2 \mathrm{~F} 1988 \& \_$alid $=1629$ 550564>. Acesso em: 22 ago. 2009. doi: 10.1016/00448486(88)90029-4.

WATANABE, W.O. et al. Production of fingerling Florida red tilapia (Tilapia hornorum X T. mossambica) in floating marine cages. Progressive Fish-Culturist, v.52, p.158-161, 1990. Disponível em: <http://afsjournals.org/doi/pdf/10.1577/15488640\%281990\%29052\%3C0158\%3APOFFRT\%3E2.3.CO\%3B2>. Acesso em: 12 jul. 2009. doi: 10.1577/1548-8640.

WEBSTER, C.D. et al. Effect of protein level and feeding frequency on growth and body composition of cage-reared channel catfish. Progressive Fish-Culturist, v.54, p.92-96, 1992. Disponível em: <ht tp://afsjournals.org/doi/pdf/10.1577/15488640\%281992\%29054\%3C0092\%3AEOPLAF\%3E2.3.CO\%3B2>. Acesso em: 12 jul. 2009. doi: 10.1577/1548-8640. 